\title{
A Rare Case of Multiple Scar Contractures after Burn Injury $^{*}$
}

\section{Haibin Wang, Zhongsheng Sun, Xiang Xu, Shengkang Luo}

Department of Cosmetic and Plastic Surgery, The Second People’s Hospital of Guangdong Province, Guangzhou, China. Email: "luoshengkang63@hotmail.com

Received February $18^{\text {th }}, 2013$; revised March $31^{\text {st }}, 2013$; accepted April $7^{\text {th }}, 2013$

Copyright (C) 2013 Haibin Wang et al. This is an open access article distributed under the Creative Commons Attribution License, which permits unrestricted use, distribution, and reproduction in any medium, provided the original work is properly cited.

\begin{abstract}
A nine-year-old girl sustained extreme postburn contractures of the face, neck, both axillae, elbows, wrists, and ankles, due to flame injury 5 years ago. No primary and plastic surgical burn treatment was available in a remote area of China. From October, 2005 to April, 2007, all adhesions were released in five operations and the huge defects covered with local musculo-cutaneous flaps, z-plasties, and with thick split skin gafts. This led to an optimal functional result and an aesthetic restoration of the face, giving her, back her self-esteem in daily life.
\end{abstract}

Keywords: Burn; Scar; Contracture

\section{Case Report}

Physical Examination: 1) lower eyelid ectropion, epiphora, and compulsive bow posture; 2) eversion of the lower lip caused by mandible-chest adhesion; 3) contracture of perioral scars, leading to the impossibility of mouth opening, closing, and drooling; 4) the cervicomental and mandible angles had completely disappeared; 5) the left and right rotation and particularly the extension of the neck were severely restricted and were classified grade IV [1] cervico-thoracic adhesion; 6) The wide scar over the chest fused with both axillary scars inhibiting the abduction of the upper arms; 7) severe scar contractures over both wrists and fingers; 8) scar contractures over the dorsum of both feet, hindering their walking functions (Figures 1-4).

\section{Operations}

1) Under general anesthesia, the patient was laid supine on the operating table with the shoulders padded below to make the neck extended. With further tumescent epinephrine-mediated vasoconstriction, a semicircular incision was made deep to the platysma muscles of the anterior neck, and the chin was lifted to get the head fully extended. Then, blunt dissection was carried out to the surface of the hyoid bone to restore a natural cer-

${ }^{*}$ Conflicts of interest: None declared; Funding: None; Ethical approval: Not required.

"Corresponding author. vico-mental angle. After the release of the lateral scar contractures, the head could be fully extended and rotated.

To cover the exposed jawbone and reshape the chin, a flap consisting of scar tissue, the platysma muscle, and the submuscular fascia with the width narrowed between both facial arteries, the pedicle inferior to the chin was raised. The lower margin of this composite flap was su-
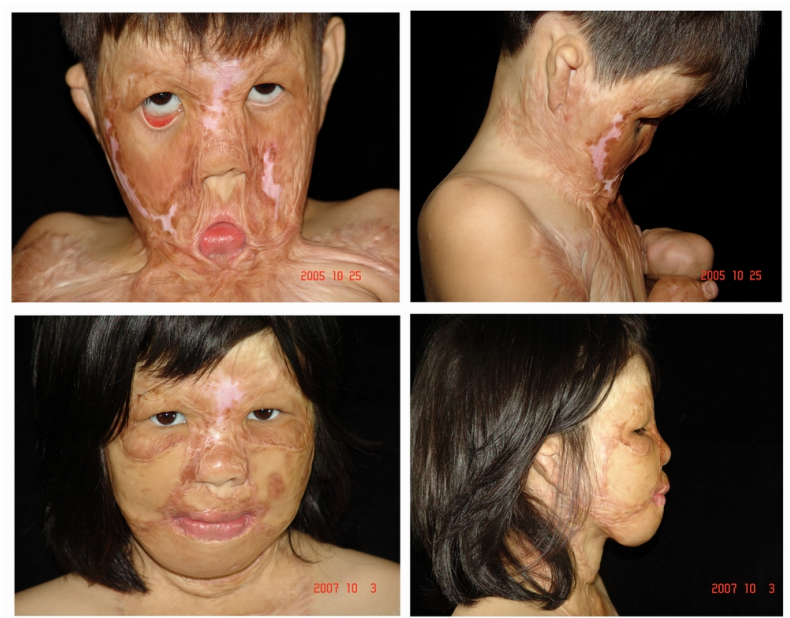

Figure 1. Pre-surgical condition of neck five years after the burn accident (the upper two); the last surgery the nose had a normal shape and the lips could be closed the ectropion of the right lower eyelid is corrected, and removal of the circular scar contracture around the nose (the down two). 

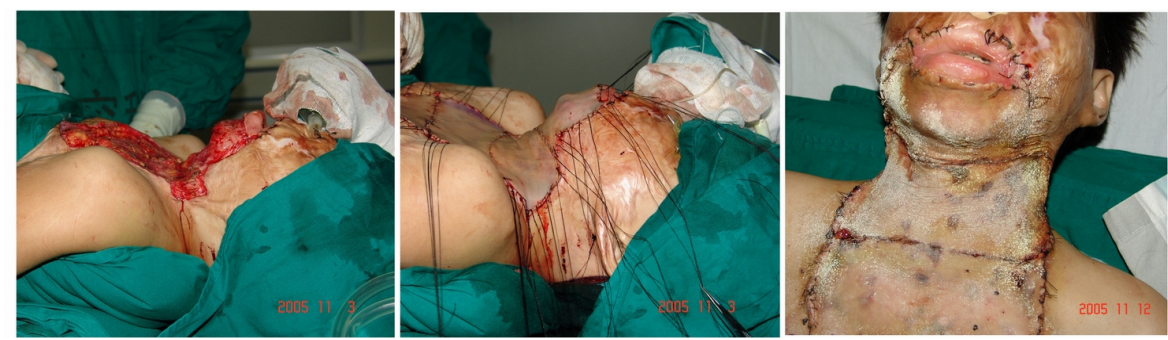

Figure 2. In the first operation, the neck adhesions were released and the huge defect grafted with thick split skin from the back.
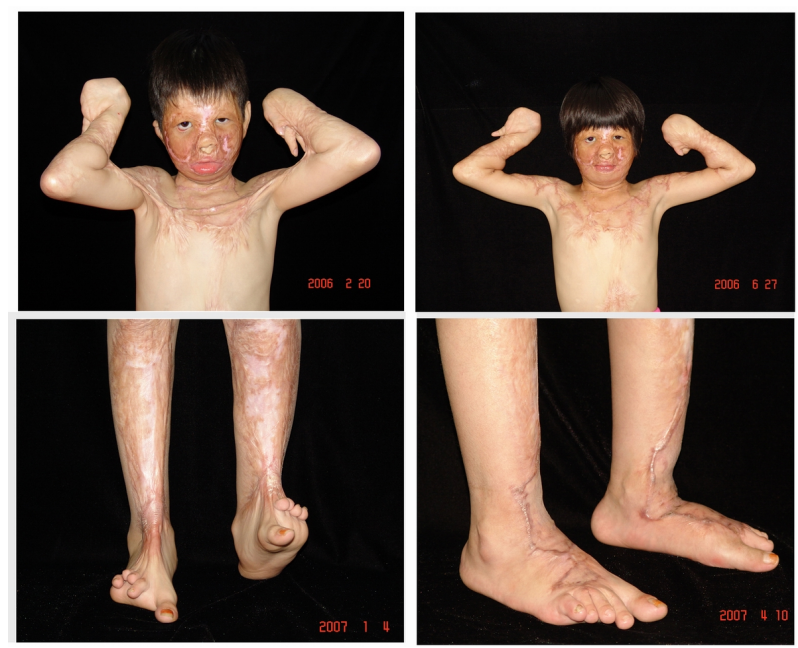

Figure 3. After releasing of the axillary scar contractures and split skin grafting, both arms could be lifted up (the upper two); after releasing of scar contractures at the dorsum of both feet and defect coverage by thick split skin grafts, walking was no problem (the down two).

tured to the mentolabial sulcus to amplify the chin, and deepen the cervicomental angle as well.

A circular scar contracture between the oral commissures and the hyoid bone was excised, which fully released the oral commissures and corrected the microstoma. After complete scar excision and release, a defect measuring $30 \times 18 \mathrm{~cm}$ extending from the oral commissures to the mid chest was grafted with four sheets of thick split skin harvested with an electrical dermatome from the back (Figure 2).

2) Three months later, the girl underwent a second operation for release of the axillary scar contractures and split skin grafting under intravenous anesthesia and thoracic epidural anesthesia. The postoperative mobile range of the shoulder joints was improved greatly, with the abduction reaching 90 degree on both sides (Figure 3).

3) Another three months later, the girl underwent the third operation for release of the scar contractures at the wrists and dorsum of both hands by thick split skin graft. After surgery, the functions of the wrists were fully restored, and all fingers could be fully extended and abducted (Figure 4).
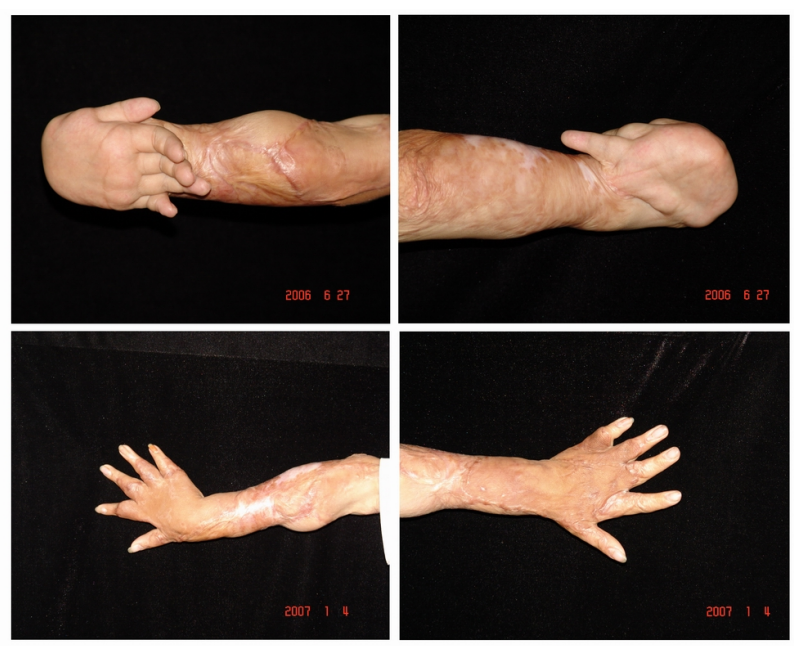

Figure 4. After releasing the scar contractures at the wrists and dorsum of both hands by thick split skin graft, the functions of the wrists were fully restored, and all fingers could be fully extended and abducted.

4) Another three months later, the girl received the forth operation for release of scar contractures at the dorsum of both feet and defect coverage by thick split skin grafts under continuous epidural anesthesia, with the functions of ankles excellently restored postoperatively (Figure 3).

5) The girl received the last surgery another six months later for scar release to correct the ectropion of the right lower eyelid, and removal of the circular scar contracture around the nose (Figure 1).

\section{Discussion}

There are many approaches available to release the scar contracture of the neck [2-4], including full-thickness or split thickness skin graft, local flaps, free flaps, and tissue expansion. This child needed multiple operations to release the scar contractures at the cervico-thoracic region, the axillae, wrists and ankles, which led to limited flaps to choose from. When it comes to grade III-IV contracture of the neck, many of the abovementioned methods have its shortcomings: the donor site of the locoregional flap is often damaged and cannot provide enough 
tissue coverage. A free flap is often too bulky even after thinning procedures, and finding a suitable donor vessel in badly scarred tissue is a challenge in technique and expertise, especially in children. Prefabricated and preexpanded flaps require multiple staged surgical procedures demanding a high degree of technical skills and nursing. For these reason, and with a limited tolerance of this girl we decided to take thick split skin grafts from the back the traditional as the first choice to solve the scar contractures over the neck, chest, shoulders and four limbs of the girl.

The advantages of thick split skin grafts are as follows: 1) it is a convenient skin source, which can cover the largest defects; 2) it is similar to full thickness skin graft and is sufficiently resistant and elastic, and less prone to re-contracture; 3 ) it has a high take rate, is not bulky, and is similar in texture; 4) it does not hinder the neck mobility, facilitating rehabilitation and functional restoration.

The cervico-mental angle is not only a key part of the physiological curvature of the neck, but also the pivotal point of the flexion and extension of the neck. All scar tissues in the groove between the hyoid bone and thyroid cartilage should be radically excised to expose the cervico-mental angle. The lower end of the flaps should not extend beyond the cervico-mental angle to assure its depth but should be located over the salivary gland.

When thick split-skin grafts are applied, they should be secured to the wound bed by multiple horizontal mattress sutures. At the site of the cervico-mental angle, the graft should be secured to the wound bed by a continuous horizontal suture, and two mattress sutures are set at the upper and lower edges of the thyroid cartilage, attaching the skin grafts closely to the bed, and preventing a possible necrosis caused by gliding of the skin grafts during swallowing [5]. Postoperatively, a chin strap and a neck collar from foam rubber must be worn to fix neck and chin for the next six months. The patients are encouraged to perform over-extension of the neck and massage the grafts vertically to prevent secondary contractures [6].

\section{REFERENCES}

[1] W. Wei, "Plastic Surgery, ” Zhejiang Science and Technology Press, Hangzhou, 1999, p. 826.

[2] I. Kuran, T. Turan, B. Saadikoglu, et al., "Treatment of a Neck Burn Contracture with a Super-Thin Occipito-Cervico-Dorsal Flap,” Burns, Vol. 25, No. 1, 1999, pp. 88-92. doi:10.1016/S0305-4179(98)00130-2

[3] R. Ogawa, H. Hyakusoku, I. Iwakiri, et al., "Severe Neck Scar Contracture Reconstructed with a Ninth Dorsal Intercostal Perforator Augmented 'Super Thin Flap',' Annals of Plastic Surgery, Vol. 52, No. 2, 2004, pp. 216219.

[4] M. Rashid, M. Zia-Ul-Islam, S. U. Sarwar, et al., "The Expansile' Supraclavicular Artery Flap for Release of Post-Burn Neck Contractures," Journal of Plastic, Reconstructive \& Aesthetic Surgery, Vol. 59, No. 10, 2006, pp. 1094-1101. doi:10.1016/j.bjps.2005.12.058

[5] J. G. McCarthy, "Plastic Surgery," 3rd Edition, W. B. Saunders Co., Philadelphia, 1990, pp. 2063-2068.

[6] S. Bhattacharya, S. K. Bhatnagar and R. Chandra, "Postburn Contracture of the Neck-Our Experience with a New Dynamic Extension Splint,” Burns, Vol. 17, No. 1, 1991, pp. 65-67. doi:10.1016/0305-4179(91)90016-A 